Supporting information

\title{
Elastic marine biodegradable fibers produced from poly $[(R)-3$-hydroxybutylate-co- 4-hydroxybutylate] and evaluation of their biodegradability
}

Taku Omura $^{1}$, Katsuya Komiyama ${ }^{1}$, Akira Maehara ${ }^{2}$, Taizo Kabe ${ }^{3}$, Tadahisa Iwata ${ }^{1 *}$

${ }^{1}$ Graduate School of Agricultural and Life Sciences, The University of Tokyo, 1-1-1 Yayoi, Bunkyo-ku, Tokyo 113-8657, Japan

${ }^{2}$ Niigata Research Laboratory, Mitsubishi Gas Chemical Co., Inc., 182, Tayuhama, Kita-ku, Niigata, Niigata 950-3112, Japan)

${ }^{3}$ Japan Synchrotron Radiation Research Institute, 1-1-1, Koto, Sayo-cho, Sayo-gun, Hyogo 679-5198, Japan

*Corresponding author. E-mail: atiwata@mail.ecc.u-tokyo.ac.jp; Tel: +81-3-5841-5266

Table S1. Fractionation of $\mathrm{P}(3 \mathrm{HB}-\mathrm{co}-16 \mathrm{~mol} \%-4 \mathrm{HB})$ with chloroform $/ \mathrm{n}$-hexane.

\begin{tabular}{|c|c|c|c|c|c|c|c|c|c|}
\hline $\begin{array}{l}\text { Sample } \\
\text { fraction }\end{array}$ & $\begin{array}{c}\text { Conc. of } n \text {-hexane } \\
\qquad / \text { vol\% }\end{array}$ & $\begin{array}{l}\text { amt. of sample in } \\
\text { fraction / wt } \%\end{array}$ & $\begin{array}{c}\text { 4HB content } \\
\text { / mol\% }\end{array}$ & $M_{\mathrm{w}} \times 10^{5}$ & PDI & \multicolumn{2}{|c|}{$\begin{array}{c}T_{\mathrm{m}} \\
\rho^{\circ} \mathrm{C}\end{array}$} & $\begin{array}{l}\Delta \mathrm{H}_{\mathrm{m}} \\
/ \mathrm{Jg}^{-1}\end{array}$ & $\begin{array}{l}T_{g} \\
/ \quad{ }^{\circ} \mathrm{C}\end{array}$ \\
\hline Original & - & 100 & 16.3 & 6.6 & 2.9 & 102.8 & 168.0 & 2.2 & -6.9 \\
\hline 1 & 50 & 1.3 & 3.1 & 10.7 & 2.2 & 173.0 & - & 63.6 & -0.18 \\
\hline 2 & 52 & 3.3 & 5.2 & 9.4 & 2.7 & 137.0 & 162.0 & 37.6 & -5.04 \\
\hline 3 & 54 & 1.2 & 9.5 & 9.2 & 1.0 & 136.8 & 161.5 & 36.6 & -4.39 \\
\hline 4 & 56 & 1.3 & 7.8 & 10.5 & 2.2 & 135.7 & 160.4 & 47.7 & -3.06 \\
\hline 5 & 58 & 69.3 & 16.3 & 8.6 & 1.9 & - & - & - & -6.96 \\
\hline
\end{tabular}




\begin{tabular}{|c|c|c|c|c|c|c|c|c|c|}
\hline 6 & 60 & 16.6 & 17.0 & 5.0 & 2.3 & - & - & - & -7.4 \\
\hline 7 & 62 & 3.89 & 17.7 & 1.4 & 1.3 & - & - & - & -9.2 \\
\hline
\end{tabular}

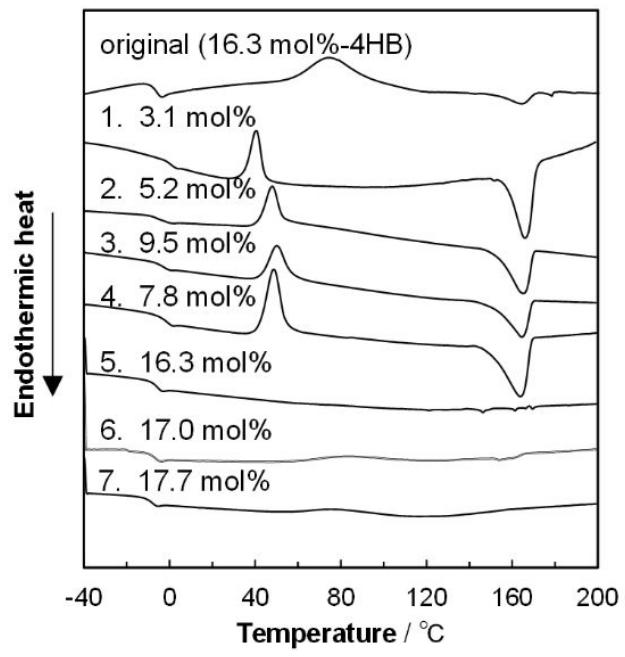

Fig. S1. Second heating scans of DSC curves of the original and fracted samples. 


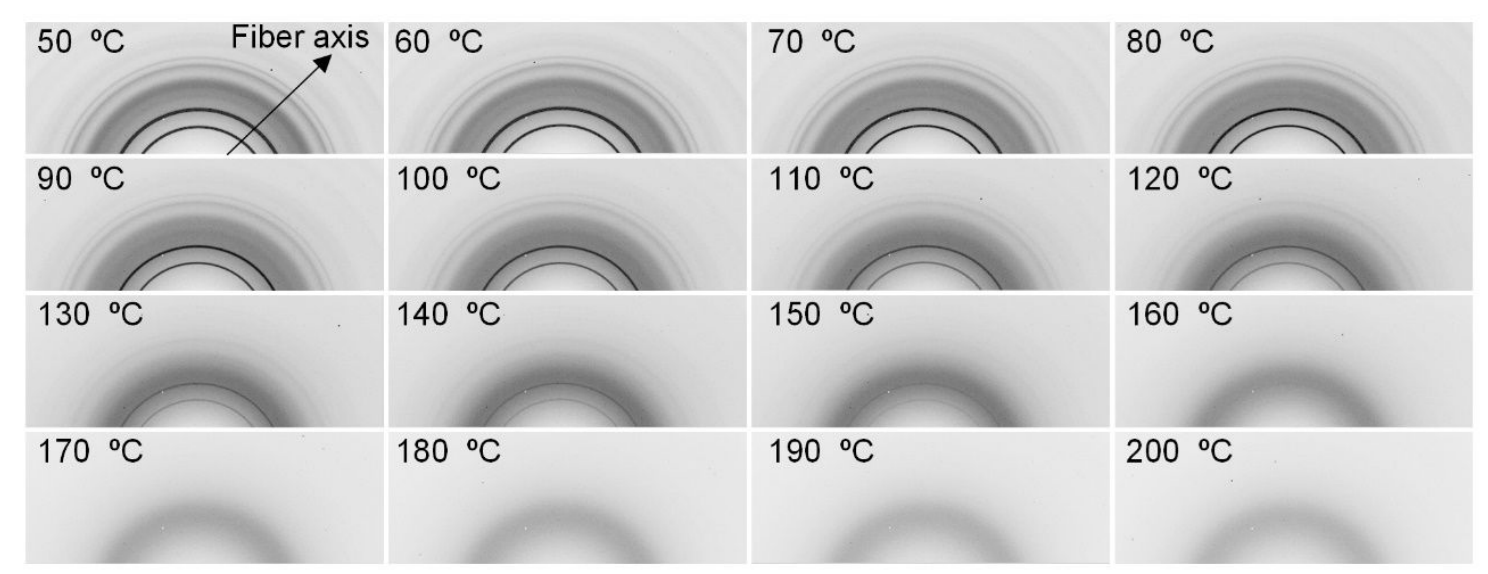

Fig. S2. 2D-WAXD patterns of $\mathrm{P}(3 \mathrm{HB}-\mathrm{co}-16 \mathrm{~mol} \%-4 \mathrm{HB})$ at a heating rate of 10

${ }^{\circ} \mathrm{C} /$ min.
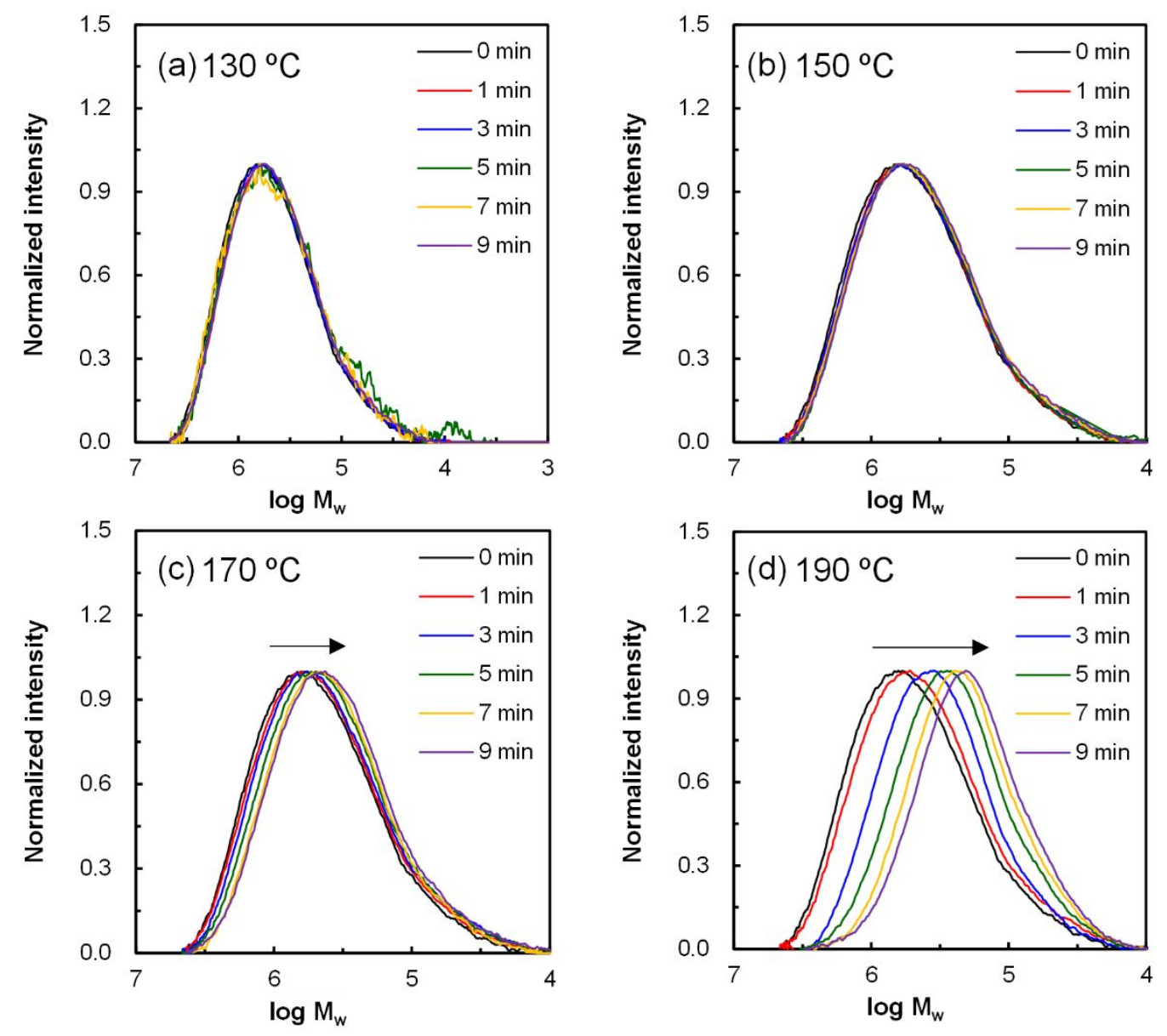
Fig. S3. Changes in GPC profiles of $\mathrm{P}(3 \mathrm{HB}-c o-16 \mathrm{~mol} \%-4 \mathrm{HB})$ during melt-spinning at (a) $130{ }^{\circ} \mathrm{C}$, (b) $150{ }^{\circ} \mathrm{C}$, (c) $170{ }^{\circ} \mathrm{C}$, and (d) $190^{\circ} \mathrm{C}$.

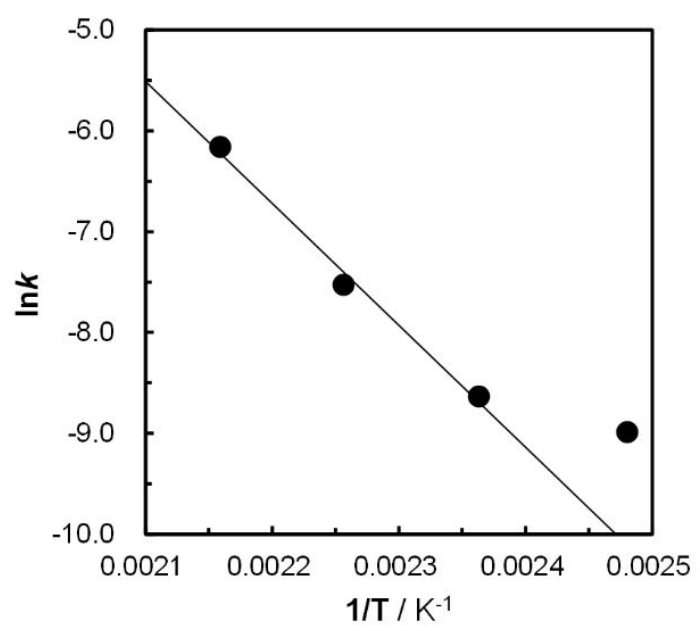

Fig. S4. Arrhenius plot describing the thermal degradation of $\mathrm{P}(3 \mathrm{HB}-\mathrm{co}-16 \mathrm{~mol} \%-4 \mathrm{HB})$ during melt-spinning. 


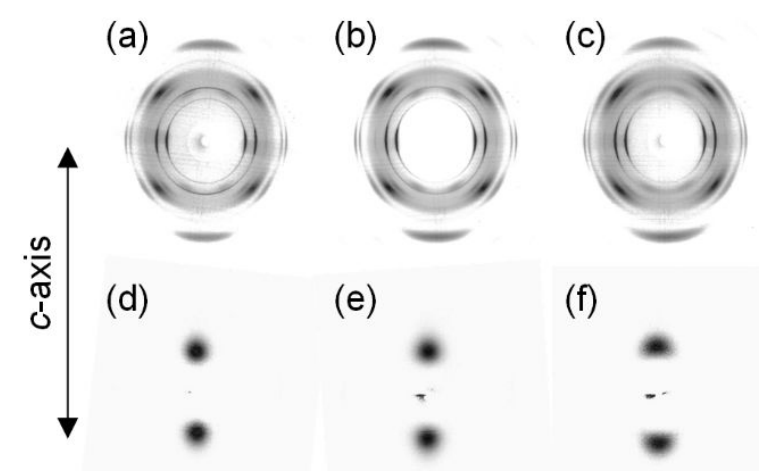

Fig. S5. 2D-WAXD and -SAXS patterns of $\mathrm{P}(3 \mathrm{HB}-$ co-16 mol\%-4HB) fibers melt spun at (a and d) $130{ }^{\circ} \mathrm{C}$, (b and e) $150{ }^{\circ} \mathrm{C}$ and (c and f) $170{ }^{\circ} \mathrm{C}$. 